\title{
Derecho y nanotecnología: definiendo lo invisible
}

\author{
Law and nanotechnology: defining the invisible \\ Direito e nanotecnología: definir o invisível \\ Droit et nanotechnologie: définir l'invisible
}

\author{
Ricardo Santana Cabello \\ Magíster de Derecho \\ Universidad Pontificia Bolivariana, Colombia. \\ Universidad de Deusto, España. \\ Correo electrónico: ricardo.santanacabello@upb.edu.co \\ Piedad Gañán Rojo \\ Doctora en ingeniería de materiales \\ Universidad Pontificia Bolivariana \\ Correo electrónico: piedad.ganan@upb.edu.co
}

Cómo citar este artículo:

Santana, R. \& Gañán, P. (2019). Derecho y nanotecnología: definiendo lo invisible. Revista de la Facultad de Derecho y Ciencias Políticas, 49 (131), pp. 507-520. doi: http://dx.doi.org/10.18566/ rfdcp.v49n131.a11

Recibido: 27 de septiembre de 2016

Aprobado: 26 de abril de 2018. 


\section{Resumen}

El presente trabajo constituye una aproximación a la nanotecnología desde el punto de vista jurídico; se presentan y analizan diferentes definiciones de nanomaterial acogidas -especialmente de la Unión Europea y la Organización Internacional de Normalización- a fin de estudiar la pertinencia de la definición dada por el Consejo Nacional Asesor de la Red Colombiana de Nanociencia y Nanotecnología.

\section{Palabras Clave}

Derecho tecnológico, Protección de Consumidores, Nanomaterial, Nanotecnología.

\section{Abstract}

The present paper constitutes an approach to nanotechnology from a legal perspective. Different recognized nanomaterial definitions - especially by the European Union and the International Organization for Standardization - are presented and analyzed, with the aim of studying the suitability of the definition given by the Consejo Nacional Asesor de la Red Colombiana de Nanociencia y Nanotecnología (National Advisory Council of the Colombian Network of Nanoscience and Nanotechnology).

\section{Key Words}

Technology law; consumer protection; nanomaterial; nanotechnology.

\section{Resumo}

Esse artigo constitui uma aproximação à nanotecnologia desde o olhar jurídico; apresentam-se $\mathrm{e}$ analisam-se diferentes definições de nanomaterial - conforme a União Europeia e a Organização Internacional de Normalização - a fim de estudar a pertinência da definição dada pelo Conselho Nacional Assessor da Rede Colombiana de Nanociência e Nanotecnologia.

\section{Palavras chaves}

direito tecnológico, proteção de consumidores, nanomaterial, nanotecnologia. 


\section{Résumé}

Ce présent article constitue une approche à la nanotechnologie depuis le point de vue juridique ; on y présente et analyse différentes définitions de nanomatériel en usage, particulièrement celle de l'Union Européenne et de l'Organisation international de Normalisation- afin d'étudier la pertinence de la définition donnée par le Conseil National Consultatif du Réseau Colombien de Nanoscience et Nanotechnologie.

\section{Mots clés}

Droit technologique, Protection des consommateurs, Nano matériel, Nanotechnologie.

\section{Introducción}

Con la intención de arrojar luz sobre el concepto de nanotecnología inicialmente se realiza una aproximación etimológica: la palabra nanotecnología está compuesta de nano, que proviene de la palabra griega vóvos (MoralesLuckie \& Guadarrama-Reyes, 2015) y que hace referencia a enano o diminuto. Por tanto se trata de mejoras en determinados procesos a un nivel pequeño o diminuto. En concreto, la nanoescala es la referencia. En el Sistema Internacional de Unidades se trata de un prefijo que alude a $10^{-9}$ metros $\left(10^{-9} \mathrm{~m}\right)$. Como ejemplo se podría señalar que un nanómetro es con respecto a un metro, lo que el diámetro de un balón de fútbol es respecto al diámetro del planeta Tierra. El motivo por el que existe el interés en manipular los materiales en dicha escala es porque se presentan propiedades muy diferentes a una escala micro o macro. Esto se debe a que se rigen por leyes de la mecánica cuántica, presentando propiedades y aplicaciones muy diferentes para una amplia gama de sectores productivos. Sin embargo, existen vacíos de conocimiento científico sobre el comportamiento de dichos nanomateriales, por lo que en algunos países se han aprobado regulaciones específicas para proteger los derechos de los consumidores. En el presente estudio se realiza una exploración de la definición de nanomaterial propuesta por ISO (International Organization for Standardization), como punto de referencia para comprender la situación de Europa al respecto. Las conclusiones extraídas podrán ser aplicadas a la definición de nanomaterial acogida en el contexto colombiano. Se concluye que está alineada a las definiciones internacionales y al actual estado del arte, aunque debe ser constantemente revisada para que permita la función de salvaguardar la seguridad pública. 


\section{Normas ISO}

En primer lugar se deben destacar las normas de ISO correspondientes a la nanotecnología, es decir, de la organización de mayor importancia en la promoción de estándares internacionales regulatorios. Desde 1946 ISO ha promovido más de 21.000 estándares que han cubierto aspectos de manera transversal en la industria. La nanotecnología es un campo destacado de su producción en los últimos años.

En el año 2005 se creó el Comité Técnico ISO/TC 229, con la intención de normalizar el campo de la nanotecnología. Se trata de "el entendimiento y control de la materia y procesos a nanoescala, típicamente, pero no exclusivamente, por debajo de los 100 nanómetros [...] y utilizar las propiedades de los materiales a nanoescala [...] para crear materiales mejorados, metodologías, modelaje y simulaciones" (ISO, 2019). En dicho comité, 37 países participan; de Sudamérica: Colombia, Perú y Brasil. Por su parte, Argentina figura como país observador.

El Comité Técnico ISO/TC 229 publicó su primer resultado concreto el 15 de agosto de 2008. Fue la Especificación Técnica ISO/TS 27687 (ISO, 2008), donde se acogía una lista de definiciones y términos relacionados con las partículas en el campo de la nanotecnología. Hoy día es una norma internacional retirada -fase con código 95.99 desde el 4 de junio de 2015-, puesto que fue revisada por la Especificación Técnica ISO/TS 80004-1 (ISO, 2010) ${ }^{1}$ y por la ISO/TS 80004-1 (ISO, 2015). En esta última norma se recoge el concepto de nanoescala de la ISO/TS 80004-1 de 2010, que incluye un tamaño aproximado entre 1 y $100 \mathrm{~nm}$; así como el nanoobjeto: "objeto que presenta una, dos o tres dimensiones externas en nanoescala” (ISO, 2010).

La definición establecida en la norma ISO/TS 80004-1 (desde ahora, Norma ISO) es una definición que está inspirando numerosas normativas en diferentes latitudes. Sin embargo, no se puede asegurar que sea una definición científica pacífica, principalmente por los retos que sigue afrontando la nanotecnología. El motivo es que si bien en el rango $1 \mathrm{~nm}-100 \mathrm{~nm}$ determinados materiales presentan una fenomenología única, así como efectos cuánticos como las olas de De Broglie, Ley de Coulomb, Efecto Túnel; o propiedades fisicoquímicas

$1 \quad$ Adoptada de manera idéntica en el contexto colombiano a través de ICONTEC, tras la aprobación de la Norma Técnica Colombiana 6149 de 15 octubre de 2015. 
diversas, en ocasiones, estos fenómenos también se dan cuando las dimensiones externas sobrepasan el margen superior de los $100 \mathrm{~nm}$.

Por consiguiente, se puede encontrar justificación en la palabra “aproximado”, puesto que dependerá del material. En ese rango se presentan típicamente los fenómenos característicos de la nanoescala, de ahí que desde un punto de vista investigativo sea acertado. Además, desde el punto de vista normativo, este método de clasificación presenta la ventaja de incluir un gran número de materiales, en caso de que se quiera tomar como referencia para una normativa más conservadora. Además, no excluye definiciones posteriores para subdividir grupos de nanomateriales en normativas específicas de acuerdo con su evaluación de riesgo.

\section{Unión Europea}

En el caso de la Unión Europea, el punto de arranque de la institucionalización fue la Comunicación de la Comisión Europea, publicada el 12 de mayo de 2004 (COM(2004)338) (EC, 2004). En ésta se propone el desarrollo responsable de la nanotecnología, a partir del compromiso ético debido a los riesgos derivados para la salud, seguridad humana, así como del medio ambiente. De la misma manera, se alienta el desarrollo y aplicación de una regulación ajustada, mediante la participación de agentes sociales.

En el apartado 3.4.4 se establece que es "esencial” una pronta y apropiada regulación nanotecnológica de los campos del derecho medioambiental, protección del consumidor y salud pública; además se hace especial hincapié en la tutela de los derechos de los trabajadores, consumidores e inversores (EC, 2004). Con todo, en aras de evitar una potencial inflación normativa, se propuso el uso de la normativa vigente en aquel momento, así como su revisión para ir ajustándola a las tecnologías que estaban por llegar.

Por otro lado, en la misma Comunicación se puede rescatar la aproximación al concepto de "nanotecnología”, que es definida en los siguientes términos: "Nanotecnología se refiere a la ciencia y tecnología, a nanoescala, de átomos y partículas, así como a los principios científicos y nuevas propiedades que pueden ser observadas y aprovechadas en una escala micro o macro" (EC, 2004). A pesar de esta aproximación, no se incluyó la definición de nanomaterial. Paralelamente, se instó a la investigación y construcción científica de conceptos técnicos. Dicho debate continúa en la actualidad, caracterizado por 
una considerable complejidad técnica. La Comisión Europea, años más tarde, propuso una definición a pesar de la posición del Parlamento Europeo, expresada a través de la Resolución de 24 de abril de 2009 (EP, 2009), que sugería que no se continuase promoviendo iniciativas legislativas nanotecnológicas hasta que no existiese consenso sobre las definiciones más básicas (Brosset, 2013).

La Comisión Europea inició un procedimiento acelerado de consulta al Comité Científico sobre Riesgos Emergentes e Identificados de Salud (SCENIHR). Para lograr una adecuada gestión del procedimiento se estableció una fecha límite y unos parámetros claros a fin de satisfacer las necesidades de definición (Brosset, 2013). La Comisión Europea, ante la demora de semanas, basándose en la información obtenida de los reportes previos, propuso la definición de nanomaterial, a través de la Recomendación 2011/696 (EC, 2011). Fue la siguiente:

Por (nanomaterial) se entiende un material natural, secundario o fabricado que contenga partículas, sueltas o formando un agregado o aglomerado y en el que el 50 \% o más de las partículas en la granulometría numérica presente una o más dimensiones externas en el intervalo de tamaños comprendido entre $1 \mathrm{~nm}$ y $100 \mathrm{~nm}$. En casos específicos y cuando se justifique por preocupaciones de medio ambiente, salud, seguridad o competitividad, el umbral de la granulometría numérica del $50 \%$ puede sustituirse por un umbral comprendido entre el $1 \%$ y el $50 \%$ (EC, 2011).

En la misma Recomendación se incluyó que en diciembre de 2014 se revisaría tal definición: “a la luz de la experiencia adquirida y de la evolución científica y tecnológica. Conviene que la revisión se centre principalmente en la cuestión de si debe aumentarse o reducirse el umbral de la granulometría numérica del 50 \%” (EC, 2011). Producto de esta disposición, el Joint Research Centre of the European Commission (JRC) comenzó a desarrollar una serie de informes técnicos para proponer mejoras científicas sobre nanotecnología. Se utiliza el tercer informe (JRC, 2015) publicado en junio de 2015, para revisar la definición de la Comisión Europea y las diferentes opciones para modificarla.

En primer lugar se planteó la posibilidad de incluir el factor del origen de los materiales para clasificarlos como nanomateriales ${ }^{2}$. Como se puede observar,

2 Por ejemplo, Francia, a través del Decreto No. 2012-232 del Ministerio de Ecología, del Desarrollo sostenible, de los Transportes y de la Vivienda acoge la definición de nanopartícula únicamente como la que es producto de manufactura intencional (Camacho et al., 2016). 
según la definición de la Comisión Europea se incluyen los materiales naturales, secundarios o fabricados. El informe se decanta por no discriminar según el origen. El motivo expuesto es que es más razonable realizar una clasificación con un criterio bien establecido y, posteriormente, en caso de acogerse por las diferentes regulaciones europeas, adaptar la definición y hacer las provisiones legales específicas necesarias. Además, no existe evidencia de una posible diferencia entre las propiedades que presenta un nanomaterial natural con respecto al mismo nanomaterial manufacturado (JRC, 2015).

La segunda cuestión de relevancia es que la definición se limitó al material particulado; por tanto, se aparta del criterio de la Norma ISO. Según el Informe, la inclusión en la definición de material no particulado cambiaría el objeto de la definición actual; sería una concepción diferente y conllevaría a un necesario rediseño de la misma. Por consiguiente, se desaconsejó la inclusión de tales materiales (JRC, 2015). Otro motivo por el que la Comisión Europea y los comités técnicos se han decantado por centrarse en los materiales nanoparticulados a la hora de construir el concepto de nanomaterial, se debe a que el informe presentado de SCENIHR (SCENIHR, 2010) confirmaron mayor probabilidad de exposición al público de este tipo de materiales (JRC, 2015) por lo que presentaban mayor valor regulatorio. Es destacable que elementos de la definición encuentran justificación desde el punto de vista regulatorio, a pesar de que el mismo informe establece que se debe llegar a una definición independientemente de la regulación. La idea subyacente del informe es que posteriormente cada regulación debe hacer los ajustes necesarios.

En tercer lugar, dado que el tamaño es la única característica común de los nanomateriales, desde los primeros informes presentados por los diferentes comités científicos se consideró que el tamaño de las partículas debía ser el principal factor de referencia (JRC, 2015). Además, este enfoque se encuentra alineado con otras definiciones en el ámbito internacional, como la Norma ISO: entre $1 \mathrm{~nm}$ y $100 \mathrm{~nm}$ que, a pesar de que no incluye toda la fenomenología dable a nanoescala, sí considera la mayor parte. El informe también desaconseja criterios adicionales al tamaño, como pueden ser propiedades presentadas por los nanomateriales debido a que dependerá del tipo de material y de sus propiedades específicas (JRC, 2015). En caso de acogerse tales criterios, debería hacerse con cautela y tras la elaboración de una guía clara de cómo aplicarlos.

Por consiguiente, se debe precisar que se estableció para las nanopartículas el rango de $1 \mathrm{~nm}$ a $100 \mathrm{~nm}$, sin la cautela de que sea un rango de referencia - como sucede con la Norma ISO - y no rígido. Es decir, no se incluyó la 
palabra "aproximadamente" en el momento de definir tal rango, lo que no significa necesariamente que no pueda aproximarse en el momento en que diferentes Estados miembros de la Unión Europea e instituciones, públicas y privadas, acojan y apliquen tal definición. Sin embargo, tal vaguedad en la norma conlleva que esté expuesta tanto a interpretaciones extensivas como restrictivas, es por esto que la finalidad de armonizar dicha definición puede no ser tan efectiva.

En cuarto y último lugar, es relevante la inclusión de un factor cuantitativo como el 50\% de las partículas en la granulometría numérica con dimensiones externas en escala nanométrica. Sin embargo, la misma definición incluye un elemento de flexibilidad, para no excluir los nanomateriales con menos del $50 \%$ de nanopartículas y que aun así interese clasificarlos como nanomateriales, debido a su potencial dañino para el medio ambiente, la salud, la seguridad o la competitividad.

Esto puede llevar a que, por ejemplo, se desarrolle un material con el 49\% de partículas en la granulometría numérica con dimensiones externas de $2 \mathrm{~nm}$ y, por tanto, se presenten atributos propios de la nanoescala. Sin embargo, al aplicar la definición de la Comisión Europea se llegaría a la conclusión de que no es un nanomaterial si no tiene afectación del medio ambiente, salud, seguridad o competitividad. En caso de modificarse el porcentaje siempre se encontrarían casos fronterizos no considerados nanomateriales; por esto el informe (JRC, 2015) confirma que elegir el porcentaje, en este caso el $50 \%$, es una cuestión meramente política. Además la afectación del medio ambiente, la salud, la seguridad o la competitividad puede conllevar efectos contraproducentes, puesto que para "justificar" tales preocupaciones en los campos mencionados, en muchos casos se debe realizar ensayos previos de dichos nanomateriales. Esto lleva a la conclusión de que durante tal ensayo no se sabe si se está analizando un nanomaterial, ya que se determinará ex post.

En cuanto a la justificación de la preocupación en los campos mencionados es interpretable. Por ejemplo, una justificación laxa sin estudios determinantes o al menos estudios que evidencien el posible riesgo. Sin embargo, tal interpretación no debería ser aplicada, ya que si posteriormente se descarta tal interés en los campos mencionados, se le desclasificaría como nanomaterial; esto restaría seguridad y eficiencia al desarrollo de la nanotecnología. Otra forma de interpretarlo sería restrictiva, por lo que si no está claramente justificada la afectación a dichos campos, no se podrá clasificar como nanopartícula. Esto conllevaría a que numerosos materiales con componentes a nanoescala, no 
fuesen clasificados como nanomateriales y, por tanto, no serían subsumibles en presupuestos de hecho de las normas específicas para nanomateriales, produciendo resultados no deseados. Por consiguiente, en este momento se deben aunar esfuerzos para establecer unos conceptos claros y así poder ofrecer seguridad. El hecho de dejar un margen de interpretación tan amplio, dada la cantidad de agentes que la pueden aplicar, resta aceleración al proceso de normalización.

Ahora bien, una Recomendación Europea no se trata de una regulación vinculante. Los Estados se podrán apartar del contenido de la misma y otorgar una definición diferente. Se trata de una guía para que los Estados la acojan o se inspiren; pero no tiene porqué conllevar una consecuencia legal: "Las recomendaciones permiten a las instituciones dar a conocer sus puntos de vista y sugerir una línea de actuación sin imponer obligaciones legales a quienes se dirigen" (EU, 2019). Es por esto que el texto afirma: "Se invita a los Estados miembros, las agencias de la Unión y los operadores económicos a utilizar la siguiente definición del término nanomaterial cuando adopten y apliquen legislación, políticas y programas de investigación sobre productos de nanotecnologías" (EC, 2011).

Se trata de la aplicación del método soft law, a través del cual se armoniza la materia dejando un margen a los Estados para adaptarla en contraposición del hard law, que uniformiza mediante una normativa vinculante para los Estados miembros. Por ejemplo, con otro instrumento legislativo como el Reglamento Europeo, de aplicación erga omnes, directa y de manera íntegra. Dada la importancia y la repercusión de este tema, la aplicación de un Reglamento Europeo que incluya una definición única de referencia de nanomaterial debería ser el objetivo a conseguir. Ello es justificado, especialmente, por su posible incidencia en la salud pública.

\section{Colombia}

En el contexto colombiano, la definición de nanomaterial no es un tema que haya pasado desapercibido. La Red Colombiana de Nanociencia y Nanotecnología, desde su nacimiento en septiembre de 2014, ha seguido de cerca el desarrollo de estas nuevas tecnologías y las aproximaciones regulatorias en otras latitudes. Para lograr estos desarrollos, dicha red creó el Consejo Nacional Asesor, integrado por 10 científicos y académicos expertos, provenientes de universidades y de centros de investigación nacionales. En el 
momento en que se crea, se da una situación de urgencia, ya que en Colombia eran y son muchos productos de fácil acceso para el consumidor que incluyen nanomateriales, a pesar de que no existe una regulación específica. Tras un periodo de estudio y discusión, dicho Consejo propone la siguiente definición de nanomaterial:

Material natural o artificialmente sintetizado, manufacturado o fabricado que exhibe propiedades, fenómenos o efectos biológicos que son atribuibles a sus dimensiones hasta una escala límite de un micrómetro. En el caso específico de materiales nanoparticulados, se consideran así cuando estos presenten una distribución mayor al $10 \%$ de partículas inferiores o iguales a $100 \mathrm{~nm}$ en al menos una de sus dimensiones. Además de esta clasificación se incluyen los materiales no necesariamente nanoparticulados pero que han sido modificados a escala nanométrica en su conformación o interfase para dar lugar a un material con nuevas propiedades (Camacho et al., 2016).

En primer lugar, el rango de fenomenología se extiende a un micrómetro, esto es muy significativo, porque se incluye un criterio no tanto del tamaño sino de los fenómenos atribuibles a ese tamaño. Esta primera parte de la definición es ambiciosa y ese es el motivo por el cual se desaconsejaba en el Informe (JRC, 2015) abarcar el concepto de nanomaterial según la fenomenología de los materiales, debido a que podrían variar de manera significativa tales propiedades según el material en cuestión. Justamente, prever toda esa información es un reto hasta el día de hoy especialmente para un rango tan amplio.

Esta norma coincide con los criterios aplicados por la American Food and Drug Administration (FDA) mediante la Guía para la Industria que publicó en junio de 2014, dice así:

1. Material manufacturado o terminado con al menos una dimensión en nanoescala -aproximadamente $1 \mathrm{~nm}$ a $100 \mathrm{~nm}-$;

2. Material manufacturado o terminado que exhibe propiedades o fenomenología, incluyendo propiedades físicas o químicas o efectos biológicos que son atribuibles a sus dimensiones, incluso si esas dimensiones sobrepasan el rango de nanoescala, hasta un micrómetro" (FDA, 2014).

Esta definición también añade que si se trata de materiales nanoparticulados se consideran como tales cuando presenten una distribución mayor al 10\% de partículas con dimensiones menores o iguales a $100 \mathrm{~nm}$ (Camacho et 
al., 2016). De la misma manera que sucede con la redacción de la definición propuesta por la Comisión Europea, resta la duda de si se trata de una escala aproximada o no. Es decir, si se trata de un material nanoparticulado y el $35 \%$ de las partículas tienen dimensiones externas de $110 \mathrm{~nm}$ ise debería considerar nanomaterial, si es que presenta propiedades, fenomenología o efectos biológicos típicos de una escala inferior a la micro? La respuesta debería ser positiva, puesto que la escala hasta $100 \mathrm{~nm}$ debe ser un rango no taxativo, para poder abarcar en la definición los materiales que en definitiva presentan atributos propios de la escala menor a la micro. Además la FDA aclara que el hecho de que el nanomaterial sea mayor a la micra no significa que no pueda manifestar propiedades diferentes y que dependan del tamaño del mismo (FDA, 2014).

Es relevante que la distribución sea del $10 \%$ a diferencia del $50 \%$ comentado previamente en el contexto europeo. De esta manera, siempre se dará la posibilidad de que se den casos fronterizos; sin embargo, prever un 10\% hace que se abarquen más materiales y que, por tanto, la definición sea más amplia. Como se decía en el mismo informe europeo, es una cuestión política decidir el porcentaje. Además, este asunto ha generado una polémica significativa dado que el Parlamento Europeo manifestó en su Resolución sobre el Reglamento Delegado de la Comisión, de 12 de diciembre de 2013, sobre la información alimentaria facilitada al consumidor sobre la definición de nanomaterial artificial (EC, 2013), en el considerando 13, que se sugiere disminuir el umbral al $10 \%$, puesto que el $50 \%$ en la distribución de partículas con un tamaño de $1 \mathrm{~nm}$ a $100 \mathrm{~nm}$ puede ser demasiado alto. A continuación se presentan las consideraciones sobre un ajuste a la definición de nanomaterial:

M. Considerando que, de acuerdo con dicha Recomendación de la Comisión, en casos específicos y cuando se justifique por preocupaciones de medio ambiente, salud, seguridad o competitividad, el umbral de la granulometría numérica del $50 \%$ puede sustituirse por un umbral comprendido entre el $1 \%$ y el $50 \%$;

N. Considerando que, de acuerdo con el dictamen del CCRSERI, para la identificación de un nanomaterial podría utilizarse un valor umbral del $0,15 \%$ sobre la base de un enfoque estadístico;

O. Considerando que la Autoridad Europea de Seguridad Alimentaria, en consulta con su comité científico y con la red para los nanomateriales en los alimentos y en los piensos, informó a la Comisión, el 3 de octubre de 2012, de que, a la vista de las actuales incertidumbres en relación con la seguridad, sería oportuno considerar un umbral numérico inferior para las 
nanopartículas, por ejemplo del $10 \%$, para las aplicaciones relativas a los alimentos, en lugar del que se propone actualmente en la Recomendación (del $50 \%$ );

P. Considerando que, según las conclusiones preliminares de la reunión del Consejo de Administración de la ECHA, el Consejo de Administración y el Director Ejecutivo de la ECHA opinan que la información actualmente disponible sobre nanomateriales es insuficiente para juzgar adecuadamente su impacto potencial, y que es necesario mejorar esta situación por lo que respecta al objetivo de proteger la salud humana y el medio ambiente;

Q Considerando que, de acuerdo con un estudio representativo sobre la percepción de la opinión pública respecto de los nanomateriales, realizado por el Instituto Federal Alemán para la Evaluación del Riesgo (BfR) en 2008, los nanomateriales en alimentos registran la menor aceptación por parte de la opinión pública;

R. Considerando que, según la propuesta de Reglamento relativo a los nuevos alimentos, presentada por la Comisión, la definición de (nanomaterial artificial») del Reglamento sobre la información alimentaria facilitada al consumidor se aplicaría asimismo a los nuevos alimentos;

S. Considerando que, a luz de los dictámenes del CCRSERI, la EFSA y la ECHA y del estudio del BfR, el uso de nanomateriales en los alimentos puede suscitar preocupación con respecto a su seguridad;

T. Considerando que, por consiguiente, el umbral del $50 \%$ propuesto por la Comisión es contrario al objetivo fundamental de la Directiva de perseguir un alto nivel de protección de la salud y los intereses de los consumidores, y que el umbral del 10 \% recomendado por la EFSA sería más apropiado, si está sujeto a revisión;

1. Presenta objeciones al Reglamento Delegado de la Comisión, de 12 de diciembre de 2013, por el que se modifica el Reglamento (UE) nº 1169/2011 del Parlamento Europeo y del Consejo, sobre la información alimentaria facilitada al consumidor, en lo relativo a la definición de (nanomaterial artificial (EC, 2013).

Otro comentario necesario es que el rango en el caso de los materiales nanoparticulados no se establece de $1 \mathrm{~nm}$ a $100 \mathrm{~nm}$; sino que es menor que $100 \mathrm{~nm}$. Ello incluye las partículas menores a $1 \mathrm{~nm} \mathrm{y}$, por tanto los fullerenos, los copos de grafeno y los nanotubos de carbono de pared simple con una o más dimensiones externas inferiores a $1 \mathrm{~nm}$; que deben ser considerados nanomateriales con propósitos regulatorios. 


\section{Conclusiones}

El concepto de nanomaterial se caracteriza por su complejidad técnica y científica. Se han hecho muchas aproximaciones a nivel internacional; sin embargo, no existe una definición científica completa que pueda servir de paradigma. A pesar de esto, hay definiciones estructuradas, como la de la Comisión Europea o la Norma ISO, que dicen mucho sobre los avances en esta materia y cómo se está trabajando y delimitando poco a poco dicha definición. Además sirve como referencia para instrumentos legislativos que dependiendo del objeto regulado puede ser adaptada. En Colombia, el Consejo Nacional Asesor de Nanociencia y Nanotecnología ha propuesto una definición alineada con los presupuestos internacionales, aunque existan partes interpretables por lo que se debe asegurar una adecuada implementación en políticas y legislación. Igualmente, se recomienda revisar dicha definición en los próximos años, a medida que se disponga de mayor información y probablemente las definiciones internacionales hayan convergido.

\section{Referencias}

Brosset, E. (2013). The Law of the European Union on Nanotechnologies: Comments on a Paradox. Reciel. Review of European, Comparative and International Environmental Law, 22(2), 155-162. http://doi.org/10.1111/reel.12030

Camacho, Á., Duarte, Á., Dubay, D., Forero, E., Gónzalez, E., Jaramillo, F., ... Urquijo, W. (2016). Definición de Nanomateriales para Colombia. Colomb. Quim., 45, 15-20.

EC. (2004). Towards a European strategy for nanotechnology.

EC. (2011). Recomendación de la Comisión Europea.

EC. (2013). Reglamento Delegado (UE) No 1363/2013.

EP. (2009). European Parliament resolution on regulatory aspects of nanomaterials.

EU. (2019). Reglamentos, directivas y otros actos legislativos.

FDA. (2014). Considering Whether an FDA-Regulated Product Involves the Application of Nanotechnology.

ISO. (2008). Especificación Técnica ISO/TS 27687.

ISO. (2010). Especificación Técnica ISO/TS 80004-1.

ISO. (2015). Especificación Técnica ISO/TS 80004-1.

ISO. (2019). Comité Técnico ISO/TC 229. 
JRC. (2015). Towards a review of the EC Recommendation for a definition of the term "nanomaterial."

Justo-Hanani, R., \& Dayan, T. (2014). The role of the state in regulatory policy for nanomaterials risk: Analyzing the expansion of state-centric rulemaking in EU and US chemicals policies. Research Policy, 43(1), 169-178. http://doi.org/10.1016/j. respol.2013.06.008

Morales-Luckie, R. A., \& Guadarrama-Reyes, S. C. (2015). Nanotecnología, el gran mundo de lo realmente pequeño (donde el tamaño sí importa). Journal of Chemical Information and Modeling. http://doi.org/10.1017/СBO9781107415324.004

SCENIHR. (2010). Scientific Basis for the Definition of the Term "nanomaterial." 